\title{
Factors Associated With Work Ability in Patients Undergoing Surgery for Cervical Radiculopathy
}

Eunice Ng, Venerina Johnston, Johanna Wibault, Hakan Lofgren, Asa Dedering,

Birgitta Öberg, Peter Zsigmond and Anneli Peolsson

\section{Linköping University Post Print}

\section{Tweet}

N.B.: When citing this work, cite the original article.

Original Publication:

Eunice Ng, Venerina Johnston, Johanna Wibault, Hakan Lofgren, Asa Dedering, Birgitta Öberg, Peter Zsigmond and Anneli Peolsson, Factors Associated With Work Ability in Patients Undergoing Surgery for Cervical Radiculopathy, 2015, Spine, (40), 16, 1270-1276.

http://dx.doi.org/10.1097/BRS.0000000000001010

Copyright: Lippincott, Williams \& Wilkins

http://www.lww.com/

Postprint available at: Linköping University Electronic Press

http://urn.kb.se/resolve?urn=urn:nbn:se:liu:diva-125696 
Work ability and Cervical radiculopathy

\title{
FACTORS ASSOCIATED WITH WORK ABILITY IN PATIENTS UNDERGOING SURGERY FOR CERVICAL RADICULOPATHY
}

\author{
Abstract \\ Study Design. Cross-sectional study \\ Objective. To investigate the factors associated with work ability in patients undergoing \\ surgery for cervical radiculopathy.
}

Summary of Background Data. Surgery is a common treatment for cervical radiculopathy in people of working age. However, few studies have investigated the impact on the work ability of these patients.

Methods. Patients undergoing surgery for cervical radiculopathy $(n=201)$ were recruited from spine centres in Sweden to complete a battery of questionnaires and physical measures the day before surgery. The associations between various individual, psychological and workrelated factors and self-reported work ability were investigated by Spearman rank correlation coefficient, multivariate linear regression and forward stepwise regression analyses. Factors that were significant $(\mathrm{p}<0.05)$ in each statistical analysis were entered into the successive analysis to reveal the factors most related to work ability. Work ability was assessed using the Work Ability Index (WAI).

Results. The mean WAI score was 28 (SD 9.0). The forward stepwise regression analysis revealed six factors significantly associated with work ability which explained $62 \%$ of the variance in the WAI. Factors highly correlated with greater work ability included greater selfefficacy in performing self-cares, lower physical load on the neck at work, greater selfreported chance of being able to work in six months' time, greater use of active coping strategies, lower frequency of hand weakness and higher health-related quality of life. Conclusions. Psychological, work-related and individual factors were significantly associated with work ability in patients undergoing surgery for cervical radiculopathy. High 
self-efficacy was most associated with greater work ability. Consideration of these factors by surgeons pre-operatively may provide optimal return to work outcomes after surgery. Key Words: Work ability, Work Ability Index, neck pain, cervical radiculopathy, selfefficacy

\section{INTRODUCTION}

Anterior Cervical Decompression and Fusion (ACDF) and Posterior Cervical Foraminotomy (PCF) surgery are common treatments for cervical radiculopathy. ${ }^{36}$ Despite generally favourable outcomes in $80 \%$ of cases, ${ }^{6,22,23,25}$ there are reports of residual long-term deficits in self-reported function, general health and neck muscle strength and endurance in patients undergoing surgery for cervical radiculopathy. ${ }^{6,10,23,25}$ A six year follow-up after ACDF reported that $50 \%$ of patients continued to be on sick leave. ${ }^{26}$ Given the frequency of surgery $^{19}$, the number of patients undergoing surgery who are of working age, ${ }^{19}$ the risks accompanying invasive surgery, ${ }^{2}$ the relatively lengthy recovery period, ${ }^{2}$ and the long-term disability ${ }^{6,10,23,25}$ for some patients, it is reasonable to expect some impact on work-related function and ability to return to work. Prolonged absence from work due to neck problems can cause substantial financial hardship, exclusion from the labour market and social isolation. ${ }^{13,32}$ Research regarding work ability in patients undergoing surgery for cervical radiculopathy has attracted little attention. In fact, the authors were unable to locate studies which used a validated scale to assess the work ability for this population. 1,7,10,24,26 Identifying the factors associated with work ability would be helpful in reducing work limitations in patients undergoing surgery for cervical radiculopathy. 
The purpose of this study was to understand the factors associated with work ability measured by the Work ability index (WAI) in patients undergoing surgery for cervical radiculopathy.

\section{MATERIALS AND METHODS}

\section{Study design and population}

This cross-sectional study obtained data from 201 (Table 1) patients with cervical radiculopathy scheduled to undergo elective surgery at four spine centres in southern Sweden during 2009 to 2012. Inclusion criteria were patients between 18 to 70 years undergoing surgery for cervical disc disease. Two approaches were used in this study with $81 \%$ undergoing an ACDF and 19\% receiving a PCF involving one to four cervical segmental levels. Suitability for surgery was based on several criteria: clinical findings determined by a spine surgeon, Magnetic Resonance Imaging (MRI) confirming the presence of disc herniation with or without osteophytes, or a stenosis caused by osteophytes and persisting cervical radiculopathy for at least two months. Exclusion criteria were a history of fracture or subluxation of the cervical column, the presence of previous trauma or injuries to the neck, specific pathologies (e.g. myelopathy, congenital cervical abnormalities, malignancy or spinal tumour, spinal infection), previous cervical spine surgery, systemic disease (such as fibromyalgia, generalised myofascial pain or rheumatoid arthritis), persisting or recurring severe back pain with disability and/or neurological signs, a diagnosed psychiatric disorder, known drug abuse and limited familiarity with the Swedish language. All data was gathered with a questionnaire administered the day before surgery.

\section{Dependent Variable - Work ability}


The work ability index (WAI) was used to assess the individual's ability to work. ${ }^{11}$ This selfreport scale is a summary measure of seven items addressing current work ability compared with lifetime best (scored 0-10), work ability in relation to work demands (scored 2-10), number of current diseases diagnosed by a physician (scored 1-7), estimated work impairment due to diseases (scored 1-6), sick leave during the past 12 months (scored 1-5), self-perceived prognosis of work ability two years from now (scored 1-7) and mental resources for work evaluated with three questions (scored 1-4). An overall WAI score ranged from 7-49, with a higher score indicating better work ability. Developers of the WAI recommended total scores be categorized into four levels; poor (7-27), moderate (28-36), good (37-43) and excellent (44-49) when used as an outcome measure in intervention studies. ${ }^{28,33}$ The validity and reliability of the WAI have been found to reliably predict work disability, retirement and mortality. ${ }^{11}$

\section{Independent Variables}

\section{Individual factors}

These included smoking status (yes/no), level of physical activity (1=inactivity, 4=high activity), use of pain medication (1=several times a day, 4=never), whether living alone and the presence of children living at home. Patients rated the severity of their neck pain, headache and dizziness at rest or during movements on a $100 \mathrm{~mm}$ visual analogue scale (VAS), ranging from 0 (no symptoms) to 100 (worst symptom severity). Self-reported frequency of neck pain, neck stiffness, shoulder pain, arm pain, hand weakness, problems lifting the arms, headache, back pain and problems sleeping were assessed on a scale of 1 (symptoms experienced all the time) to 5 (never experienced symptoms). 
Self-reported general health was evaluated with the reliable five dimension European quality of life scale (EQ5D and EQ VAS) with higher scores (EQ5D ranged -0.624 to 1 and EQ VAS ranged 0 to 100 ) indicative of better quality of life. ${ }^{3}$

The 10-item Neck Disability Index (NDI) ${ }^{21}$ was used to evaluate self-perceived disability in everyday life due to neck pain. Each item was scored from 0 (no disability) to 5 (high disability) with total scores expressed as a percentage. ${ }^{35}$ Larger scores represent a higher level of disability.

\section{Psychological factors}

The frequency of being worried or anxious about symptoms was scored on a five-point scale of 1 (felt worried or anxious all the time) to 5 (never felt worried or anxious). Symptom satisfaction was measured on a seven point scale of 1 (happy about symptoms) to 7 (unhappy about symptoms).

Self-efficacy in performing 20 everyday functional tasks in spite of pain was evaluated using the self-efficacy scale (SES) anchored from 0 (not at all confident) to 10 (very confident). ${ }^{4}$ Total scores range from 0 to 200 with higher scores indicating higher perceived selfefficacy. $^{4}$

The Modified Zung scale evaluated self-rated depression with a standardised 23-item questionnaire. ${ }^{14}$ Each item is scored from 1 (rarely or none of the time) to 4 (most of the time). Total scores range from 20 to 80 and are categorised into normal, mildly, moderately and severely depressed. 
The Modified somatic perception questionnaire (MSPQ) was included to assess the presence of heightened autonomic or somatic awareness in those with chronic pain or disabilities. ${ }^{18}$ The 22-item self-report questionnaire helps identify somatic complaints that may be related to psychological responses of anxiety or depression. Each question was scored on a four point scale from 0 (not at all) to 4 (extremely/could not be worse). The summary of all points are converted into the final score using an algorithm ranging from 0 to 39. A higher score indicates a greater risk of somatization.

Cognitive and behavioural strategies adopted to cope with pain were evaluated with the Coping Strategy Questionnaire (CSQ). ${ }^{29}$ There are 50 items in total, 48 of which evaluated six subscales of cognitive coping strategies (ignoring pain, diverting attention, catastrophising, praying/hoping, reinterpreting pain and coping self-statements) and one subscale of a behavioural coping strategy (increasing pain behaviours). ${ }^{29}$ Patients rated each coping subscale from 0 (never do that) to 6 (always do that) with a maximum score of 36 for each subscale.

\section{Work-related factors}

These included current working status, the amount of time worked per week, work satisfaction, workplace satisfaction and whether they had changed their work due to neck problems were assessed as a dichotomous outcome (yes/no). Participants rated the physical load on the neck due to work on a three item scale of mild, moderate or heavy. Participants estimated their chance of being able to work in six months' time on a five item scale (1=very small, 5=very large).

\section{Statistical Analysis}


Parametric and non-parametric descriptive statistics (depending on the variable) were used to describe the patient group. The dependent variable, WAI was retained as a continuous variable to enable regression modelling. However, the number and percent of scores within each of the four WAI categories will be presented. To examine the association between individual, work-related and psychological factors and the WAI, correlation and regression analyses were conducted. Bivariate correlations between all factors (continuous and discrete) and the WAI score were determined by Spearman's rank correlation coefficient analysis. Those significantly correlated with the WAI $(\mathrm{p}<0.05)$ were continuous and underwent a standard linear regression analysis followed by a forward step-wise regression analysis to reveal the factors most related to the WAI. Adjusted $\mathrm{R}^{2}$, Beta, b-value, and standard error $\mathrm{b}$ were used to display results. Before conducting the multiple regression analysis, the possible multicollinearity of the independent variables was examined using the variance inflation factor (VIF) set at $\leq 0.10$ tolerance and $\geq 10 \mathrm{VIF}^{8}{ }^{8}$ Statistical analyses were conducted with SPSS version 20.0 (IBM Corporation New York, USA).

\section{RESULTS}

The mean (SD) score for the WAI as a continuous variable was 28 (9). Of those working, the work ability was poor 48 (37.8\%), moderate 49 (38.6\%), good 25 (19.7\%), and excellent for 5 (3.9\%) participants. The values for the individual, psychological and work-related variables for participants are listed in Tables 2 and 3 respectively. The discrepancy between the numbers of people answering the questions on the WAI is due to inconsistency in participant reports. There was also missing data for some survey items. 


\section{Associations}

Spearman rank correlation co-efficient analysis showed that 26 variables were significantly correlated $(\mathrm{p}<0.05)$ with the WAI including individual, psychological and work-related factors. Of these, 16 individual factors were significantly correlated with the WAI: the severity of current neck pain, current headache and dizziness at rest and during movements, the frequency of neck pain, neck stiffness, shoulder pain, arm pain, hand weakness, problems lifting the arms, headache, back pain and sleeping problems and score on the EQ5D, EQ VAS and NDI. Eight psychological factors were correlated with the WAI: symptom satisfaction, the frequency of feeling anxious or worried about symptoms, three subscales of the CSQ scale: coping self-statements, catastrophising, ignoring pain sensations and the MSPQ, Modified Zung self-rating depression and SES scores. Two significantly correlated workrelated factors included the physical work load on the neck and the self-perceived chance of being able to work in six months' time. There were no inter-correlations between these variables $(\mathrm{r}<0.8)$.

The standard multivariate linear regression analysis revealed 12 variables explained $66 \%$ of the WAI $(\mathrm{p}<0.0001)$ (Table 4). Although the frequency of hand weakness, frequency of back pain, symptom satisfaction and the score on the CSQ scale: ignoring pain sensations were not significant ( $p>0.05$ ), they were included as they contributed to the $\mathrm{R}^{2}$ value.

The final forward stepwise regression model produced six statistically significant variables explaining $62 \%$ of the variance of the WAI (Table 5). The most significant factor was the score for functional self-efficacy, which alone accounted for $33 \%$ of the final model. The other significant factors were physical work load on the neck at work, self-estimated chance 
of being able to work in six months' time, score on the CSQ: coping self-statements, frequency of hand weakness and the EQ5D score.

\section{DISCUSSION}

This study investigated the factors associated with work ability in patients undergoing surgery for cervical radiculopathy. The factors highly correlated with greater work ability included greater self-efficacy for functional tasks, lower physical load on the neck at work, higher chance of being able to work in six months' time, high use of active coping selfstatement strategy, lower frequency of hand weakness and a high health-related quality of life. These six variables explained $62 \%$ of the variance of the WAI. Two each of psychological, work-related and individual factors featured in the final model, demonstrating the multidimensional influence on work disability in patients undergoing surgery for cervical radiculopathy. This finding is consistent with previous studies with neck pain sufferers in the working population. ${ }^{9,17}$

Patients with cervical radiculopathy scheduled for surgery in this study were relatively disabled with 38\% scoring in the 'poor' category of the WAI. These patients also reported chronic pain with a mean duration of neck pain and arm pain of 32 and 27 months respectively. Most patients were working full-time despite the reported levels of pain, indicating the importance of understanding the impact on work ability of this group.

The scores on the self-efficacy scale for everyday tasks accounted for the largest proportion of variance in WAI, demonstrating that higher self-efficacy was positively associated with work ability. This result was not unexpected and consistent with the literature, which shows psychological factors are the most common indicators of prolonged disability in patients with 
chronic neck pain. ${ }^{9,16,17,37}$ In addition, high self-efficacy is an important determinant for functional outcomes and successful return to work in patients with musculoskeletal disorders. ${ }^{15}$ Several studies have shown the effectiveness of interventions targeting selfefficacy in improving work ability among patients with various chronic diseases. $5,15,20,30,31,34$ It appears that a higher level of control and ability to influence one's situation is synonymous with greater work ability.

A high coping self-statement strategy was also a significant contributor to high work ability. This is perhaps attributed to the prolonged duration, severity and frequency of symptoms which may have eroded the individual's ability to adjust to the chronic symptoms. Clinicians need to be alert to and regularly assess the patient's skills in managing with pain to ensure appropriate interventions are provided.

Physical load on the neck at work and self-estimated chance at being able to work in six months' time were the two work-related factors found to be significantly associated with work ability. These factors accounted for an additional $22 \%$ of the WAI. The majority of patients (50.7\%) reported work induced a heavy load on the neck and a high to very high chance of being able to work in six months' time (78\%). A previous study was unable to provide evidence for any relationship between physical versus non-physical work and functional disability, however, the NDI was the primary outcome measure. ${ }^{12}$ The WAI was used in the present study, possibly explaining the significance of the physical job demands. Surgeons should consider the role of the workplace and make recommendations to reduce the physical load on the neck. Assessment of the work demands by a physiotherapist may be useful to identify ergonomic interventions to reduce the physical load. 
The frequency of hand weakness and the EQ5D were the final two factors significantly associated with work ability. Frequency of hand weakness is a reflection of the radiculopathy and the EQ5D a reflection of poor general health so it was not surprising that those factors were important for work ability. Patients in this study scored both EQ5D and EQ VAS lower than in the 6 year follow-up study by Peolsson et al. ${ }^{25}$ This may be related to the pre-surgery results in the present study, not available in Peolsson et $a l^{25}$ but possibly also due to changes in the social security system forcing people to work full-time despite disability. Previous studies have reported pain intensity and smoking status to be significantly correlated with functional disability ${ }^{1,24,25,27}$ contrasting with our study of work ability. This suggests that while working is considered a functional activity, the determinants of the ability to work appear to be different to those for other functional tasks. The use of a specific scale to measure work ability and the inclusion of an extensive list of variables may have altered the importance of these variables for work ability.

The results of this research may be useful to surgeons when determining how best to support individuals undergoing surgery for cervical radiculopathy. A comprehensive assessment of the patient prior to surgery may identify areas of concern such as a low health related quality of life; low expectation of being at work in six months' time, and passive pain coping strategies. Attention to these concerns by a multi-disciplinary team prior to and after surgery may ensure successful functional outcomes for the individual.

A large number of patients and an extensive range of variables across the individual, psychological and work-related domains were included. These features enhance our confidence in the results. However, the cross-sectional design of this study does not enable cause-effect links between independent variables and the WAI. The predictive value of the 
psychological, work-related and individual variables can best be determined in a prospective study. In addition, the use of self-reported measures may result in response bias and social desirability bias and there was missing data from patients who failed to answer questions. Despite existing limitations the present study adds valuable information of work ability in patients with cervical disc disease, never presented before.

\section{CONCLUSIONS}

In conclusion, the results from this study demonstrate that psychological, work-related and individual factors are significantly associated with work ability in patients undergoing surgery for cervical radiculopathy. Specifically, self-efficacy for functional tasks is strongly positively associated with work ability. Together, surgeons may use these factors to provide a clearer indication of patient needs to guide treatment interventions for better work ability. 
Table 1. Demographics and background data of patients undergoing surgery for Cervical Radiculopathy (n=201)

\begin{tabular}{ll}
\hline \multicolumn{1}{c}{ Variables } & \multicolumn{1}{c}{ Values } \\
\hline Gender (n\%) & $96(48.0)$ \\
$\quad$ Female & $104(52.0)$ \\
$\quad$ Male & $49.9(8.5)$ \\
Age (y), mean (SD) & $81(15)$ \\
Weight (Kg), mean (SD) & $173.7(8.9)$ \\
Height (cm), mean (SD) & 163 \\
Surgeries (n) & 38 \\
$\quad$ ACDF & \\
PCF & 106 \\
Number of surgery levels (n) & 77 \\
$\quad 1$ level & 16 \\
2 levels & 2 \\
$\quad 3$ levels & $32(44.7)$ \\
$\quad 4$ levels & $27(40.1)$ \\
Duration of neck pain (months), mean (SD) & \\
Duration of arm pain (months), mean (SD) &
\end{tabular}


Table 2. Descriptive data for individual factors $(n=201)$

\begin{tabular}{ll}
\hline \multicolumn{1}{c}{ Individual Variables } & \multicolumn{1}{c}{ Values } \\
\hline Smoker (yes/no) (n\%) & $149(74.1) / 46(23.6)$ \\
Level of physical activity (n\%) & $17(8.5)$ \\
None & $79(39.3)$ \\
Low & $59(29.4)$ \\
Moderate & $35(17.4)$ \\
High & $71(35.3)$ \\
Pain medication use (n\%) & $42(20.9)$ \\
Several times per day & $55(27.4)$ \\
Every day & $23(11.4)$ \\
Seldom & $46(22.9) / 149(74.1)$ \\
Never & $92(45.8) / 103(51.8)$ \\
Living alone (yes/no) (n\%) & \\
Children living at home (yes/no) & \\
(n\%) & \\
Severity of symptoms (0-100mm & \\
VAS), mean (SD) & $56.5(24)$ \\
Current neck pain & $50.6(28.1)$ \\
Current arm pain & $24.6(29.3)$ \\
Current headache & $11.7(20)$ \\
Dizziness at rest & $22.1(25.5)$ \\
Dizziness during movement & \\
Frequency of symptoms (1-5), mean & \\
(SD) & \\
Neck pain & $4.2(1.1)$ \\
Neck stiffness & $3.9(1.2)$ \\
Shoulder pain & $3.5(1.3)$ \\
Arm pain & $3.8(1.2)$ \\
Problems lifting the arms & $2.8(1.4)$ \\
Hand weakness & $3.4(1.5)$ \\
Headache & $2.4(1.1)$ \\
Back pain & $2.5(1.2)$ \\
Problems sleeping & $3.1(1.4)$ \\
EQ5D score, mean (SD) & $0.4(0.3)$ \\
EQVAS score, mean (SD) & $46.9(21.3)$ \\
NDI\% score, mean (SD) & $42.8(14.7)$ \\
\hline
\end{tabular}


Table 3. Descriptive data for psychological and work-related variables $(n=201)$

\begin{tabular}{|c|c|}
\hline Variables & Values \\
\hline \multicolumn{2}{|l|}{ Psychological } \\
\hline Self-Efficacy Scale (SES) score, mean (SD) & $125.91(39.32)$ \\
\hline $\begin{array}{l}\text { Modified Somatic Perception Questionnaire } \\
\text { (MSPQ) score, mean (SD) }\end{array}$ & $7.93(4.9)$ \\
\hline $\begin{array}{l}\text { Modified Zung self-rating depression scale, } \\
\text { mean (SD) }\end{array}$ & $25.59(9.81)$ \\
\hline \multicolumn{2}{|l|}{ Coping Strategy Questionnaire, mean (SD) } \\
\hline ignoring pain & $13.98(7.24)$ \\
\hline diverting attention & $17.47(6.26)$ \\
\hline catastrophising & $14.26(8.18)$ \\
\hline praying/hoping & $12.58(5.82)$ \\
\hline reinterpreting pain & $5.44(6.44)$ \\
\hline coping self-statements & $13.69(6.88)$ \\
\hline increasing pain behaviours & $17.87(5.56)$ \\
\hline $\begin{array}{l}\text { Frequency of being worried about symptoms } \\
\text { mean (SD) }\end{array}$ & $2.38(1.091)$ \\
\hline Symptom satisfaction, mean (SD) & $5.77(1.38)$ \\
\hline \multicolumn{2}{|l|}{ Work-related } \\
\hline \multicolumn{2}{|l|}{ Working status (n\%) } \\
\hline Full time & $98(50.3)$ \\
\hline Part time & $38(19.5)$ \\
\hline Not working & $59(30.3)$ \\
\hline \multicolumn{2}{|l|}{ Sick leave status (n\%) } \\
\hline Full time & $45(22.4)$ \\
\hline Part time & $25(12.4)$ \\
\hline Not on sick leave & $124(61.7)$ \\
\hline Work satisfaction (yes/no) (n\%) & $143(95.0)$ / 7 (5.0) \\
\hline Work place satisfaction (yes/no) (n\%) & $143(96) / 6(4)$ \\
\hline $\begin{array}{l}\text { Changed work due to neck related problems } \\
\text { (yes/no) (n\%) }\end{array}$ & $50(27.9) / 129(72.0)$ \\
\hline \multicolumn{2}{|l|}{ Physical work load on neck (n\%) } \\
\hline Mild & $17(8.5)$ \\
\hline Moderate & $63(31.3)$ \\
\hline Heavy & $102(50.7)$ \\
\hline \multicolumn{2}{|l|}{ Chance of working in 6 months' time (n\%) } \\
\hline Very large & $81(40.3)$ \\
\hline Large & $68(33.8)$ \\
\hline Either/or & $13(6.5)$ \\
\hline Small & $6(3.0)$ \\
\hline Very small & $6(3.0)$ \\
\hline
\end{tabular}


Table 4. Linear multivariate regression demonstrating factors significantly associated with the WAI $(\mathrm{n}=127)$

\begin{tabular}{lrr}
\hline \multicolumn{1}{c}{ Factors } & $\mathbf{R}^{\mathbf{2}}$ & \multicolumn{1}{c}{$\mathbf{p}$} \\
\hline Severity of dizziness at rest & 0.66 & 0.008 \\
Frequency of & & \\
$\quad$ hand weakness & $\mathrm{p}>0.05$ \\
$\quad$ back pain & $\mathrm{p}>0.05$ \\
$\quad$ sleeping problems & 0.03 \\
Symptom satisfaction & $\mathrm{p}>0.05$ \\
EQ5D scale & 0.04 \\
Coping Strategy Scale (CSQ) & \\
- coping self-statements & 0.003 \\
Coping Strategy Scale (CSQ) & \\
- ignoring pain sensations & $\mathrm{p}>0.05$ \\
Modified Zung scale & 0.01 \\
Self-Efficacy for functional tasks (SES) & 0.004 \\
Physical work load on neck & $<0.0001$ \\
Chance of working in 6 months' time & $<0.0001$ \\
\hline
\end{tabular}


Table 5. Step-wise forward regression analysis for outcome on WAI to reveal variables most associated with WAI $(\mathrm{n}=127)$

\begin{tabular}{lcccc}
\hline \multicolumn{1}{c}{ Factors } & \multicolumn{4}{c}{ Step-wise regression } \\
& Cum adj. $\mathbf{R}^{\mathbf{2}}$ & Beta & \multicolumn{1}{c}{ b } & Standard error b \\
\hline Functional Self-Efficacy & 0.33 & 0.28 & 0.06 & 0.02 \\
Physical work load on neck & 0.49 & -0.33 & -4.20 & 0.76 \\
Chance of working in 6 months' time & 0.55 & -0.27 & -2.12 & 0.47 \\
CSQ coping self-statements & 0.59 & 0.20 & 0.29 & 0.08 \\
Frequency of hand weakness & 0.61 & -0.14 & -0.79 & 0.33 \\
EQ5D & 0.62 & -0.16 & 4.47 & 1.94 \\
\hline
\end{tabular}

